\title{
Development and design of management information system security protocol for wireless sensor network routing
}

\author{
Hai Ye $\mathrm{e}^{1, \mathrm{a}}$ \\ ${ }^{1}$ Qingyuan Polytechnic, Guangdong Qingyuan, 511510, China \\ ayehaiqyzyjs@163.com
}

Keywords: Security protocol; Management information system; Wireless sensor network; Routing protocol

\begin{abstract}
This paper firstly analyzes the advantages and disadvantages of various securities routing protocol of wireless sensor network. Secondly, this paper discusses construction of wireless sensor network on management system the security protocol. At last, this paper presents development and design of management information system security protocol for wireless sensor network routing. The experimental results show that this approach can improve efficiency of management information system security protocol effectively.
\end{abstract}

\section{Introduction}

The wireless communication network and traditional wireless sensor networks have different design requirements and technical targets. The data center and the latter are for the purpose of transferring data. The wireless sensor network because of its special application determines the following has some of the characteristics of wireless sensor networks, sensor hardware resource limited wireless sensor network node number is large; often need to invest in a specific region detection. Due to their size and cost constraints, the computing capability, the data processing ability, storage ability is very limited.

At present, the Internet, peer-to-peer network and Ad hoc network usually uses asymmetric cryptography mechanism to solve the problem of external attack, its disadvantage is the higher complexity of the algorithm, the calculation of storage volume is very large, not suitable for computing power, storage resources and limited energy of sensor nodes, which leads to many existing asymmetric cryptographic protocol is not suitable for the establishment of wireless sensor network. The existing security mechanism of wireless sensor network usually uses symmetric cryptography less complex to solve the external attacks; however, this mechanism there is a big security risk.

Wireless sensor network (WSN) is the current international concern, interdisciplinary, highly integrated knowledge of cutting-edge research field [1]. Sensor technology, MEMS, modern network and wireless communication technology, and promote the emergence and development of modern wireless sensor network. Wireless sensor networks extend the ability to obtain information, the physical information of the objective world with the transmission network connected together, provide the most direct, the most effective, and the most authentic information for the people in the next generation network. Wireless sensor network can obtain the objective physical information, has very broad application prospects.

Many types of sensors in wireless sensor network is capable of detecting, including seismic, electromagnetic, temperature, humidity, noise, light intensity, pressure, soil composition, size of moving objects, the speed and direction of the surrounding environment in a variety of phenomena. Give a broad application prospect of micro sensor technology and wireless networking technology for wireless sensor network based on MEMS. These potential applications can be summed up as: military, aviation, anti-terrorism, explosion-proof, disaster relief, environmental, medical, health, Home Furnishing, industrial, commercial and other fields. 
Another important function of the data link layer is data transmission error control. In the network there are two important error controls mode are the forward error control (FEC) and automatic repeat request (ARQ). The use of ARQ in the multi hop network is due to retransmission overhead and additional energy consumption but rarely used. Even using the FEC method, only low complexity of cyclic codes are taken into account, and the other is suitable for sensor network error control scheme is still in the exploratory stage.

Therefore, it is how to get to the local node topology information and under the conditions of limited resources to achieve a simple and efficient routing mechanism, which is a basic problem of WSN. In data centers, and the traditional routing protocols usually address as identifier and routing node based on WSN, but due to the random distribution of nodes, which is concerned with the sensing data monitoring area, rather than any specific node information, to form a data centric message forwarding path. Application of correlation and the designer needs to be tailored to each specific application requirements, specific design and adaptive routing mechanism. This paper presents and analyses development and design of management information system security protocol for wireless sensor network routing.

\section{Construction of wireless sensor network on management system the security protocol}

Wireless sensor networks are widely used in many fields of military, environmental science, health, space exploration, construction and city management. But now, . But now, some research results are all in the initial stage, the actual demand is still far distance. Overall, our country in wireless sensor network research is few, the wireless sensor network is an emerging technology, domestic and international level gap is not large, in a timely manner to carry out this study has profound influence on the future of human life of cutting-edge technology, for the whole country, the social economy will have great strategic significance. Data transmission network routing protocol cannot do without, however, the traditional routing protocol in wireless Ad Hoc networks are not suitable for wireless sensor network routing protocol, we must design new, suitable for the characteristics of wireless sensor networks.

The base station node sensor network to establish contact with the outside world, but each sensor node network is a self-organized wireless network without center. The dynamic changes in the network topology, the topology here refers to the network layer from the point of view. Embodied in two aspects, first of all, on behalf of the mobile host network topology in wireless channel on behalf of fixed-point and directed edges can increase or disappear; moreover, the network topology may be split and merge and so on.

In the process of setting up the structure of the consumption of energy has become an important content to consider. Experiments show that the multi hop data transmission network, the loss of energy than the direct transmission loss of energy. The introduction of multi hop routing for topology management and medium access control are significant. When the node is very close to sink when using the method, a direct route is very appropriate. However, if the sensors are randomly scattered in the area, then the multi hop routing will have greater advantages.

Each sensor node includes 1 or more sensors, mote and battery. The main detection sensor vibration and temperature data, mote is responsible for the data processing and transmission through the network to the base station. NRF9E5 is using radio frequency, system chip RF launched by Nordic company in Norway [2]. The use of GFSK modulation, strong anti-interference ability; support multi-point communications, data transmission rate up to 0.4 MB / S; Shock Burst has unique signal transmitting mode and signal carrier monitoring function, as is shown by equation (1).

$$
\widetilde{w}(m):=\left[w^{T}(m M), w^{T}(m M+1), \cdots, w^{T}(m M+M-1)\right]^{T}
$$


Based on DSSS, using physical layer data packets in the same format, the difference lies in the working frequency, modulation, and spread spectrum chip length and transmission rate. 915 / 868 $\mathrm{MHz}$ band is differential coding based on BPSK (binary phase shift keying), a $3.4 \mathrm{GHz}$ band with sixteen quadrature modulation. The $3.4 \mathrm{CHz}$ band has 16 different channels for the global uniform without the need to apply ISM (industrial, scientific, medical) band, using high order modulation technique can provide a transmission rate of $290 \mathrm{kbit} / \mathrm{s}$, communication delay and shorter throughput, less help to get more of the work week period.

WSANs is usually deployed in no communication infrastructure environment, each node has a certain mobility and limited energy, frequently changing network topology, and high real-time requirements for data transmission. These characteristics determine the application of self organization needs no center, and support multi hop and node by mobile Ad hoc network can meet the requirement of WSANs, as is shown by equation (2).

$$
P_{j-1} f=P_{j} f+Q_{j} f=\sum_{k} c_{k}^{j} \phi_{j k}+\sum_{k} d_{k}^{j} \psi_{j k}
$$

From the point of view of routing security maintenance and it is looking for the safest possible route to ensure the safety of network. If the routing protocol destruction messages has been tampered with, and it is so there was no security for any data packets on application layer for. This paper introduces a method called "security aware routing" (SAR), the idea is to find out the relationship between the true value and between the nodes, and then use the real value to generate a secure routing. The method to solve two problems and it is namely how to guarantee the security of information security in the data transmission path and routing protocol in. It is assumed that the two officers use AODV (Ad Hoc On Demand Distance Vector Routing, AODV) ad protocol through hoc network communication, their communication based on Bell-La security model.

Wireless sensor node, it is the processor module used for node device control, task scheduling, energy calculation, function coordination; wireless transceiver module is used to transmit data between nodes, frequency selection, receiving sensor module used for external sensors, signal conversion, energy supply module provides the necessary energy for sensor node, as is shown by equation(3).

$$
\omega_{i}=\sum_{j=1}^{m} \sqrt{\frac{q_{b_{j}}\left(y_{0}\right)}{p_{b_{j}}\left(y_{0}\right)}} \delta\left[I\left(\mathrm{X}_{i}\right)-b_{j}\right]+\sum_{j=m+1}^{M} \sqrt{\frac{q_{b_{j}}\left(y_{0}\right)}{p_{b_{j}}\left(y_{0}\right)}} d\left(\mathrm{X}_{i}\right) \delta\left[o\left(\mathrm{X}_{i}\right)-b_{j}\right]
$$

Rumor routing is suitable for application in sensor networks with less amount of data transmission, it can be regarded as a special kind of directed diffusion routing protocol. After the query message directed diffusion and flooding mechanism to determine the path to increase data transmission path optimization, when the network transmission data quantity is very few, the directed diffusion protocol overhead is too large. The basic idea is: the rumor routing sensor node event area generation (Agent) news agency.

As the sensor node's energy is limited, therefore, when the energy consumed, they will become a failure node. In this agreement, when the sensor node is detected by S F after the failure of the parent node, node parent nodes F S will delete from the parent node, if the parent node is empty, then the node $S$ proceed as follows.

A) node $\mathrm{S}$ to the neighbor node broadcasting a Req message;

B) node receives the Req message if it has been added to the routing backbone and the node $\mathrm{S}$ is not in its parent node in the collection, it is to node $\mathrm{S}$ returns a Res message, including its message distance parameter; 
C) Node $\mathrm{S}$ waiting for $\mathrm{T}$ time, it returns the message view distance parameter Res value, the minimum value of the node and distance parameters (more than 1) as the parent node and add it to their parent node set.

The main purpose of the transport layer security protocol in wireless sensor network is the use of lower level services, to provide transparent, reliable data transmission service to the upper layer. Therefore, the transport layer must implement the technology flow and congestion control, and error free, orderly, no loss, no duplicate data transmission function. The application layer is the specific applications of wireless sensor networks based on reflected, including a series of software application layer monitoring task.

\section{Application and analysis of routing protocols in Wireless Sensor Networks}

The wireless sensor node does not have a unified identity, multi hop communication method between nodes. Resource constrained nodes, to complex routing computation, can only obtain local topology information. In data centers, and it is wireless sensor network sensor data is only concerned with the monitoring area, and the network includes a plurality of nodes to the sink node of the data stream, according to the sensing data demand, data communication mode and flow paths, data centric message form.

Wireless sensor network is the integration of monitoring, control and wireless communication network system, the number of nodes is large (thousands or even tens of thousands of nodes), the distribution is more intensive; due to environmental impact and energy depletion, nodes are more prone to failure; environment interference and node failure caused by the change of the network topology; usually, most of the sensor nodes is fixed. In addition, sensor nodes have energy, processing power, storage capacity and communication capabilities are very limited. The primary design goal of traditional wireless network is to provide high quality service and high bandwidth utilization, then considering energy saving; and the primary design goal of wireless sensor networks is the efficient use of energy, as is shown in equation (4) [3].

$$
J_{F}(w)=\frac{w^{T} S_{B} w}{w^{T} S_{w} w}
$$

Information consultation (Sensor Protocols for Information sensor SPIN via Negotiation) is the first data centric routing algorithm, reduce data redundancy and energy loss through the inter node negotiation mechanism. SPIN is a routing algorithm based on negotiation mechanism of data centric, SPIN first node receives the data characteristics of a high level of abstraction, the formation of metadata node receives the data characteristics (meta-data). In forwarding the received data before, node A consult with metadata and the adjacent node B.

Because of the number of sensor nodes and node distribution changing in the survival process of wireless sensor network, the topology of the network is constantly changing, nodes may move, considering the scalability of wireless sensor networks, node joining and leaving the network topology changes. The MAC protocol should be scalable in order to adapt to the dynamic topology, distributed wireless sensor network changes, because the nodes of wireless sensor network computing power and storage capacity is limited, many nodes need to cooperate to complete a task application, so MAC protocol should be running distributed algorithm.

According to the routing protocol, routing structure, communication mode, routing strategy timing state maintenance, node identification and the delivery way more classification of routing protocols in wireless sensor network, according to the transmission process using the path number, can be divided into single path routing protocol and multi path routing protocol. Single path routing saves storage space, less data communication; multi path routing fault tolerance, robustness, and can choose an optimal route from many routes. 


\section{Development and design of management information system security protocol for wireless sensor network routing}

Directed diffusion routing protocol is the way, the basic idea is to build a virtual polar coordinate system, used to represent the actual network topology. The nodes in the network are formed by a sink node as the root of the tree ring. Each node uses root hops and angle to express. The topological structure of the whole network to form a tree, the root node is closer to the structure of the network, the overall understanding of the more. When a node does not know the route to destination node, it will transmit data packets to the root of the direction, until a node knows a route to the destination node. Because most of the data are sent to the roots, so the tree can work well in wireless sensor networks.

The structure of planar routing protocol is simple, easy realization, the effect of small scale sensor networks better, but the sensor network is the large number of nodes, the monitoring area, then using the plane routing protocol with network delay is large, the energy utilization efficiency is low. Although the hierarchical routing protocol is complicated, but it has a smaller network delay, high energy utilization rate and the advantages of self organization strong, can adapt to a wide range of regional monitoring requirements.

In order to analyze the performance of this Agreement and the existing agreement, the experiment in the same environment to achieve the LEACH and FCMA routing protocol, and compare their performance, as shown in figure 1. The analysis includes the following two parameters: a) the performance of the average delay time, the average packet delay time from the source node to the destination node, this parameter is used to test the protocol used by the routing algorithm is effective, is an important indicator of the routing protocol; b) the average energy consumption, the sensor nodes in the the average energy consumed by each cycle, this parameter is an important parameter to measure whether the efficient routing algorithm.

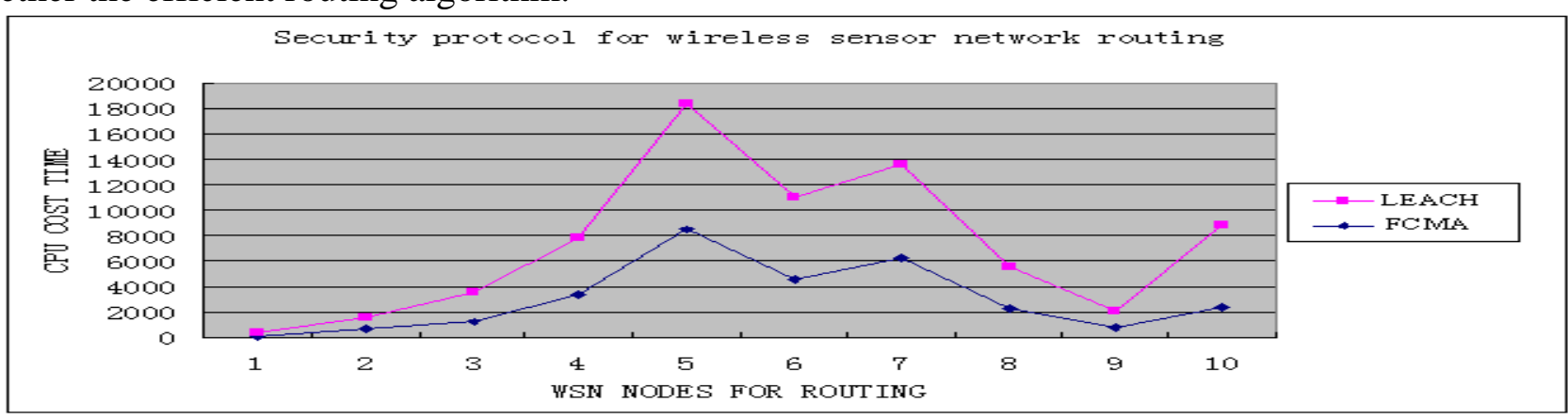

Figure. 1 Management information system security protocol for wireless sensor network routing based on LEACH with FCMA

In this paper, FCMA comes from LEACH, which is an improved version of LEACH protocol. FCMA assumes that sensor nodes in network are isomorphic and stationary. The test signal decreasing node sends energy, by detecting the response to determine the nearest neighbor node. In this way, all the nodes in the network to be able to understand each other's position, and then each node clustering according to locations to choose their own belongs, the cluster head clustering optimization to calculate the reference position of the best link node sink.

\section{Summary}

According to the research of wireless sensor network routing protocol of wireless communication line relative to the traditional routing protocols have attracted more and more people. From the above analysis we can see that each protocol is related to each other. Therefore, in a sense, it is hard to tell which more advantage of that agreement. Compared analysis of these protocols based on it. Clustering mechanism of LEACH can reduce the overall network energy consumption, prolong the survival time of the network; using the TDMA code in the cluster nodes, using the CDMA code in the cluster head 
and base station, to ensure the effective transmission of information; data acquisition and cluster head nodes are periodic, network for monitoring of continuous change events.

\section{References}

[1] K.Ramanan, E.BabuRaj, "Energy Conserved Routing Algorithm In Wireless Sensor Networks", JDCTA, Vol. 8, No. 6, pp. $01 \sim 12,2014$

[2] AL-Gabri Malek, Chunlin Li, Naji Hasan.A.H, Zhiyong Yang, Xiaoqing Zhang, "Energy Efficiency Improvement on LEACH protocol of wireless sensor networks", IJACT, Vol. 5, No. 10, pp. $1 \sim 9,2013$

[3] SU Bing, HUANG Juan, "Research on a New Energy Balancing Routing Algorithm for Wireless Sensor Networks", JCIS, Vol. 3, No. 2, pp. 42 48, 2013. 